\title{
Propionibacterium acnes-associated chronic hypertrophic pachymeningitis followed by refractory otitis media: A Case Report
}

Eiichiro Amano ( $\nabla$ eiichiroamano1122@hotmail.co.jp )

Tsuchiura Kyodo General Hospital https://orcid.org/0000-0003-0363-4853

Keisuke Uchida

Tokyo Ika Shika Daigaku - Yushima Campus

Tasuku Ishihara

Tsuchiura Kyodo General Hospital

Shinichi Otsu

Tsuchiura Kyodo General Hospital

\section{Akira Machida}

Tsuchiura Kyodo General Hospital

\section{Yoshinobu Eishi}

Tokyo Medical and Dental University

\section{Case Report}

Keywords: Propionibacterium acnes, otitis media, pachymeningitis, mastoiditis, dura mater

Posted Date: February 6th, 2019

DOI: https://doi.org/10.21203/rs.2.305/v1

License: (9) (i) This work is licensed under a Creative Commons Attribution 4.0 International License. Read Full License

Version of Record: A version of this preprint was published at BMC Neurology on January 9th, 2020. See the published version at https://doi.org/10.1186/s12883-020-1600-3. 


\section{Abstract}

Background: Hypertrophic pachymeningitis (HP) is a rare disorder that involves localized or diffuse thickening of the dura mater. HP is associated with various inflammatory, infectious, and malignant diseases, such as rheumatic arthritis, sarcoidosis, anti-neutrophil cytoplasmic antibody-associated vasculitis, IgG4-related disorders, syphilis, tuberculosis, bacterial and fungal infections, cancer, and idiopathic diseases, when evaluation fails to reveal a cause. Among them, chronic infection with Propionibacterium acnes is a rare etiology of $\mathrm{HP}$, and its pathology remains unclear.

Case Presentation: An 80-year-old man having refractory otitis media with effusion of the right ear presented with progressive right-sided headache and nausea. Post-contrast brain magnetic resonance imaging revealed right mastoiditis and remarkable thickening of the dura mater and enhancement of pia mater extending from the right middle cranial fossa to the temporal lobe.

HP secondary to middle ear infection was suspected, and a biopsy of the right mastoid was performed. An anaerobic culture of the biopsied right mastoid showed the growth of $P$. acnes, and histopathological examination using $P$. acnes-specific monoclonal antibody (PAB antibody) revealed the infiltration of inflammatory cells with $P$. acnes. Moreover, using PAB antibody, $P$. acnes was detected in the biopsy specimen of the thickening dura mater. No granulomas were identified in either specimen. HP was resolved with long-term administration of antibiotics and steroids.

Conclusion: This is the first documentation of pathologically demonstrated chronic HP associated with $P$. acnes infection followed by refractory otitis media. This report showed that chronic latent $P$. acnes infection induces chronic inflammation, which cannot be controlled without administering steroids.

Keywords: Propionibacterium acnes, otitis media, pachymeningitis, mastoiditis, dura mater

\section{Background}

Hypertrophic pachymeningitis (HP) is a rare disorder that involves localized or diffuse thickening of the dura mater [1]. Most patients present with chronic headaches with or without other neurological manifestations such as cranial nerve palsies, cerebellar ataxia, seizures, myelopathy, and visual disturbances [2]. HP is associated with various inflammatory, infectious, and malignant diseases, such as rheumatic arthritis, sarcoidosis, ANCA-associated vasculitis, IgG4-related disorders, syphilis, tuberculosis, bacterial and fungal infections, cancer, and idiopathic' diseases, when evaluation fails to reveal a cause [3]. Among them, chronic infection with Propionibacterium acnes (P. acnes) is a rare etiology of HP [4]. P.acnes is a slow-growing, non-spore forming anaerobic gram-positive bacillus that ubiquitously resides in human skin and hair follicles [5]. Intriguingly, P. acnes can invade and persist in epithelial cells and circulating macrophages, thus inducing chronic inflammation [5]; it is considered the most implicated etiological agent for sarcoidosis in Japan because it has been isolated by bacterial culture from systemic sarcoidosis lesions at high ratios [6]. 
Regarding the association of $P$. acnes with the intracranial infections, it is one of the important pathogens responsible for postoperative meningitis and subdural empyema [7-9]. However, several cases of chronic meningitis and HP due to $P$. acnes have been reported which were not associated with neurosurgical procedures or trauma [10-12]. Moreover, in some cases, both antibiotics and the concomitant use of steroids alleviated the disease course $[13,14]$.

Here we report the first case of HP due to pathologically proven chronic $P$. acnes infection, which was resolved by long-term administration of both antibiotics and steroids.

\section{Methods}

An 80-year-old man presented with deafness and otorrhea of right ear and was diagnosed as having otitis media with effusion (OME). Myringotomy, the insertion of a ventilation tube, and administration of oral antibiotics were only partially effective; his OME had been recurrent and refractory to these treatments. Although myeloperoxidase and proteinase 3 anti-neutrophil cytoplasmic antibodies (MPO-ANCA, PR3ANCA) were negative in the blood examination, otitis media with ANCA-associated vasculitis was suspected, and prednisolone (30 mg/day) was orally administered [15]. However, because the improvement in deafness and otorrhea was transient and insufficient, prednisolone administration was stopped.

Six months later, a chronic right-sided headache emerged and gradually worsened. Eight months after the onset of OME, he suffered from nausea and severe headache and was admitted to our hospital.

Post-contrast MRI revealed right mastoiditis, remarkable thickening of the dura mater, and enhanced pia mater extending from the right middle cranial fossa to the temporal lobe (Figure 1). Cerebrospinal fluid (CSF) examination revealed an elevated cell count $\left(31 / \mathrm{mm}^{3}\right.$, mononuclear cells $\left.30 / \mathrm{mm}^{3}\right)$ and total protein levels $(91 \mathrm{mg} / \mathrm{dl})$. Repeated CSF cultures were negative, and the results of the polymerase chain reaction analyses performed on the CSF for Mycobacterium tuberculosis, Epstein-Barr virus, Cytomegalovirus, and Herpes simplex virus were also negative. CSF cytology was normal, and no oligoclonal band was detected.

Both MPO-ANCA and PR3-ANCA were evaluated again in the serum; however, the results were negative. An additional blood examination showed normal angiotensin-converting enzyme, lysozyme, and immunoglobulin G4 levels. Anti-CCP antibodies, rheumatoid factor, and anti-nuclear antibodies were not detected. Serology test results for syphilis, human immunodeficiency virus, human T-lymphotropic virus type 1, candida, and aspergillus were all negative. Mycobacterium tuberculosis was not present as per the results of the interferon-gamma release assays. $\beta D$-glucan was not detected in the blood. $C$ reactive protein levels were slightly elevated $(1.19 \mathrm{mg} / \mathrm{dl})$.

HP secondary to bacterial middle ear infection was suspected, and meropenem (MEPM) (6 g/day) and vancomycin (VCM) (2 g/day) were administered. Although the CSF examination showed decreased cell counts $\left(19 / \mathrm{mm}^{3}\right)$ and protein levels $(58 \mathrm{mg} / \mathrm{dl})$ on day 13 , the patient's headache gradually worsened, 
and a post-contrast MRI showed no improvement (Figure 2). A biopsy of the right mastoid was performed, and $P$. acnes was detected using $P$. acnes-specific monoclonal antibody (PAB antibody); culture results for $P$. acnes were also positive (Figure 3 , discussed below).

To control the post-infectious intracranial inflammation, high-dose intravenous methylprednisolone (IVMP) ( $1000 \mathrm{mg} /$ day) was administered for 5 days followed by oral prednisolone ( $45 \mathrm{mg} / \mathrm{day})$, and his headache was remarkably alleviated. Because $P$. acnes is sensitive to ceftriaxone (CTRX), MEPM and VCM were switched to CTRX ( $4 \mathrm{~g} /$ day). Although the patient received predonisolone (PSL), trimethoprimsulfamethoxazole (TMP-SMX) was also prescribed for prophylaxis against pneumocystis pneumonia [16]. PSL was stopped after 4 weeks, and CTRX was administered for 7 weeks. The post-contrast MRI showed improved dural thickening, and the patient's headache disappeared.

However, HP relapsed 4 weeks after the discontinuation of PSL and 1 week after the discontinuation of CTRX (Figure 2). The patient experienced severe headache, gait disturbance, and diplopia. Neurological examination revealed right abducent paralysis and ataxia. We performed a biopsy of the dura mater; $P$. acnes was detected using PAB antibody; however, it was not detected in the culture of the dura mater (Figure 4, discussed below). After we performed a biopsy of the dura mater, high-dose IVMP (1000 $\mathrm{mg} /$ day) was administered for 5 days, followed by oral prednisolone $(45 \mathrm{mg} /$ day). CTRX ( $4 \mathrm{~g} /$ day) was simultaneously administered for 4 weeks and then switched to oral minocycline (MINO). TMP-SMX had been concomitantly administered since high-dose IVMP was initiated. After we restarted the treatment, his symptoms gradually disappeared. PSL was tapered but had been continued with both MINO and TMP-SMX for 1 year. Even after finishing these three medications, the patient has remained free from relapses for more than 1 year.

\section{Pathological Findings}

No granulomas were identified, but inflammatory cell infiltration was evident in both samples based on HE staining (Figure $3 a$ and g, Figure $4 \mathrm{~b}$ and $\mathrm{h}$ ). No organisms were identified by HE and PAS staining.

Based on reports and the literature, formalin-fixed and paraffin-embedded tissues of the right mastoid and dura mater were tested using immunohistochemical methods with PAB antibody [6] and mycobacterial species-specific monoclonal antibody [17], which were generated according to the protocol previously reported; appropriate controls were also used. Many small round bodies were detected by immunohistochemistry with PAB antibody in the inflammatory macrophages of both tissues (Figure $3 a$ and h, Figure $4 a$ and $h$ ). Immunoreactivity for mycobacterial species was not identified within either of the tissues (Figure 3c, Figure 4c). Immunoreactivity for $P$. acnes was more evident in the right mastoid than in the dura mater. CD4-, CD8-, or CD20-positive cells had infiltrated around the PAB-positive small round bodies (Figure $3 d-f$, Figure $4 d-f$ ). There were more CD8-positive cells in both tissues than CD4positive cells.

\section{Discussion And Conclusions}


This is a unique case of $P$. acnes-associated HP secondary to otitis media that was successfully treated by long-term administration of antibiotics and steroids. The biopsies and immunohistochemical analyses of the mastoid and dura mater showed the presence of $P$. acnes and inflammatory cell infiltration, which implied that $P$. acnes caused chronic inflammation. Although the administration of intravenous antibiotics improved the CSF findings at first, it did not alleviate the symptoms or the radiological findings; therefore, concomitant steroid use was necessary. In addition, the short-term administration of steroids resulted in the relapse of HP, and the long-term administration of antibiotics and steroids was necessary for HP remission. This clinical course implies that $P$. acnes established the chronic latent infection, which was controlled by the anti-inflammatory effects of steroids. To the best of our knowledge, no similar cases have been previously reported. $P$. acnes is a part of commensal microbiota and is generally present in human skin, hair follicles, and mucous membranes [5, 18]. It is well known for its involvement in the pathogenesis of acne vulgaris [19]; moreover, its role in various postoperative infections such as endocarditis, endophthalmitis, and central nervous system (CNS) infections has been reported [5]. In addition, P. acnes can invade and persist in epithelial cells and circulating macrophages in its intracellular latent form [5], which results in chronic inflammation such as sarcoidosis [6, 20,21].

CNS infections caused by $P$. acnes are generally associated with previous neurosurgical procedures, such as craniotomy and cerebrospinal shunt placement [7-9]. Brain abscess, subdural or epidural empyema, and shunt meningitis are common $P$. acnes-related CNS infections that are successfully treated by antibiotics, sometimes in combination with surgical interventions such as drainage and debridement [79]. By contrast, $P$. acnes causes meningitis without neurosurgical procedures in both immunocompromised [22] and immunocompetent patients [10-14]. In P. acnes infection cases without trauma or surgical procedures, the paths by which $P$. acnes invades the CNS putatively include (1) hematogenous routes via the diploic veins from the hair follicles or sebaceous glands of the scalp or (2) invasion from adjacent inflammation such as sinusitis, orbititis, and otitis media [4]. Anaerobic bacteria, including $P$. acnes, are important pathogens in otitis media [23] and are sometimes found in the cultures of chronic secretory otitis media [24]. In this case, chronic inflammation in OME created an anaerobic condition, which is advantageous for $P$. acnes colonization and biofilm production; consequently, $P$. acnes subsequently invaded the adjacent intracranial meninges.

Because $P$. acnes has often been considered a common contaminant of blood and fluid cultures, we analyzed the biopsied mastoid by immunohistochemistry with PAB antibody to exclude the possibility that the $P$. acnes in the fluid cultures from the biopsied right mastoid was a consequence of false-positive contamination. In addition, although the CSF culture did not test positive for $P$. acnes, it was successfully detected by immunohistochemistry with PAB antibody within the biopsied dura mater and mastoid.

Yang et al. reported a case of $P$. acnes-associated neurosarcoidosis identified by immunohistochemical analysis of the brain tissue using PAB antibody [25]. Contrarily, our case differs from sarcoidosis because pathological analyses did not reveal the presence of non-caseating granulomas. Furthermore, there was greater CD8-positive lymphocyte infiltration than CD4-positive lymphocyte infiltration in the specimens, which is distinct from sarcoidosis because CD4-positive lymphocytes are dominant in sarcoidosis [21]. 
The mechanism by which CD8-dominant inflammation was triggered was unclear; however, $P$. acnes antigens in the infected tissues putatively induced the cytotoxic immune response and exaggerated the inflammatory reaction, which cannot be controlled without the administration of steroids.

Although $P$. acnes-associated CNS infection can be treated with antibiotics in most cases, French et. al reported a rare case of $P$. acnes-associated meningitis responsive to steroids [14]. Conversely, Yamashita et. al reported a case of chronic meningitis that was successfully treated by steroids without antibiotics; this patient subsequently died of acute meningitis caused by $P$. acnes [13]. Although his case did not prove the presence of $P$. acnes in CSF during the treatment of chronic meningitis and it was unclear whether $P$. acnes actually caused the chronic meningitis, we should be aware that steroid administration against chronic $P$. acnes infection without antibiotics might result in a fatal outcome. Except for sarcoidosis, which has been reported to be caused by a hypersensitive Th1 immune response to $P$. acnes and can thus be treated by steroids, chronic $P$. acnes infection should be carefully observed when steroids are administered.

Notably, otitis media sometimes accompanies HP in anti-neutrophil cytoplasmic antibody (ANCA)associated vasculitis without systemic manifestations such as pulmonary and renal involvement $[1,26]$. It was difficult to discern between ANCA-associated vasculitis and chronic $P$. acnes-associated inflammation without either a histopathological examination or blood testing of ANCA in this patient. We should be aware of chronic inflammation caused by $P$. acnes for the differential diagnosis of HP accompanied by refractory otitis media.

In conclusion, we report the first known case of $P$. acnes-associated chronic HP followed by refractory otitis media. The patient was successfully treated by long-term administration of antibiotics and steroids. Further study is necessary to reveal how $P$. acnes induces chronic latent infection and inflammation in the brain and why both antibiotics and steroids are necessary to control the chronic inflammation.

\section{Abbreviations}

HP: Hypertrophic pachymeningitis; ANCA: anti-neutrophil cytoplasmic antibody; OME: otitis media with effusion; MPO: myeloperoxidase; PR3: proteinase 3; CSF: cerebrospinal fluid; PAB antibody: P. acnesspecific monoclonal antibody; IVMP: intravenous methylprednisolone; CTRX: ceftriaxone; MEPM: meropenem; VCM: vancomycin; PSL: prednisolone; TMP-SMX: trimethoprim-sulfamethoxazole; MINO: minocycline; CNS: central nervous system.

\section{Declarations}

\section{Ethics approval and consent to participate}

We confirm that we have read the journal's position on issues involved in ethical publication and affirm that this report is consistent with guidelines. As this is a case report describing clinical observations, ethics approval was waived. 


\section{Consent for publication}

Consent to publish was obtained in written form from the patient. The patient consented to the publication of this case report.

\section{Availability of data and material}

The datasets used and/or analyzed during the current study are available from the corresponding author on reasonable request.

\section{Competing interests}

The authors declare that they have no competing interests.

\section{Funding}

No financial support and sponsorship was obtained for this study.

\section{Authors' contributions}

EA and YE conceptualized and designed the study. KU and YE performed pathological analysis and evaluation. $\mathrm{EA}, \mathrm{KU}, \mathrm{TI}$, and $\mathrm{SO}$ performed data acquisition. $\mathrm{EA}, \mathrm{KU}, \mathrm{AM}$, and $\mathrm{YE}$ performed analysis and interpretation of clinical data. EA drafted the manuscript. YE critically revised the manuscript for content. All authors read and approved the final manuscript.

\section{Acknowledgments}

We thank Dr. Motomu Honjo and Dr. Keiji Honda, the otolarynogologists at the Tsuchiura Kyodo General Hospital, for performing the mastoid biopsy. We also thank Dr. Masataka Yoshimua and Dr. Shoko Fujii, the neurosurgeons at the Tsuchiura Kyodo General Hospital, for performing the dura mater biopsy. The authors would like to thank Enago (www.enago.jp) for the English language review.

\section{References}

1. Yokoseki A, Saji E, Arakawa M et al. Hypertrophic pachymeningitis: significance of myeloperoxidase anti-neutrophil cytoplasmic antibody. Brain. 2014;137:520-36.

2. Choi HA, Lee MJ, Chung CS. Characteristics of hypertrophic pachymeningitis in patients with granulomatosis with polyangiitis. J Neurol. 2017;264:724-32.

3. Kupersmith MJ, Martin V, Heller G, Shah A, Mitnick HJ. Idiopathic hypertrophic pachymeningitis. Neurology. 2004;62:686-94.

4. Nishioka R, Nakajima S, Morimoto Y, Hosoai H, Nakamura H. Hypertrophic cranial pachymeningitis with Propionibacterium acnes detected by dural biopsy. Clin Neurol. 1995;35:526-30. 
5. Leheste JR, Ruvolo KE, Chrostowski JE, Rivera K, Husko C, Miceli A, et al. acnes-driven disease pathology: current knowledge and future directions. Front Cell Infect Microbiol. 2017; doi:10.3389/fcimb.2017.00081.

6. Negi M, Takemura T, Guzman J, Uchida K, Furukawa A, Suzuki Y, et al. Localization of Propionibacterium acnes in granulomas supports a possible etiologic link between sarcoidosis and the bacterium. Mod Pathol. 2012;25:1284-97.

7. Ramos JM, Esteban J, Soriano F. Isolation of Propionibacterium acnes from central nervous system infections. Anaerobe. 1995;1:17-20.

8. Kranick SM, Vinnard C, Kolson DL. Propionibacterium acnes brain abscess appearing 10 years after neurosurgery. Arch Neurol. 2009;66:793-5.

9. Yakhkind A, Yacoub HA, Grove J, Varrato JD, Castaldo JE. Pathogenesis, clinical course and neuroradiological signs of Proprionibacterium acnes cerebritis: Case report and literature review. Hosp Pract. 2015;43:128-36.

10. Schlesinger JJ, Ross AL. Propionibacterium acnes meningitis in a previously normal adult. Arch Intern Med. 1977;137:921-3.

11. Ueunten D, Tobias J, Sochat M, Miranda C, Mulligan M, Yoshikawa TT. An unusual cause of bacterial meningitis in the elderly. Propionibacterium acnes. Arch Neurol. 1983:40:388-9.

12. Burnham JP, Thomas BS, Trevino SE, McElvania Tekippe E, Burnham CA, Kuhlmann FM. De Novo meningitis caused by Propionibacterium acnes in a patient with metastic melanoma. J Clin Microbiol. 2014;52:1290-3.

13. Yamashita R, Saito T, Otsuka M, Mizutani T, Ueki A. An autopsy case of Propionibacterium acnes meningitis preceded by a chronic recurrent meningitis. Neurol Med (Tokyo). 1994;41:125-31.

14. French RS, Ziter FA, Spruance SL, Smith CB. Chronic meningitis caused by Propionibacterium acnes. A potentially important clinical entity. Neurology. 1974;24:624-8.

15. Harabuchi Y, Kishibe K, Tateyama K, Morita Y, Yoshida N, Kunimoto Y, et al. Clinical features and treatment outcomes of otitis media with antineutrophil cytoplasmic antibody (ANCA)-associated vasculitis (OMAAV): A retrospective analysis of 235 patients from a nationwide survey in Japan. Mod Rheumatol. 2017;27:87-94.

16. Stern A, Green H, Paul M, Vidal L, Leibovici L. Prophylaxis for Pneumocystis pneumonia (PCP) in nonHIV immunocompromised patients. Cochrane Database Syst Rev. 2014; doi:10.1002/14651858.CD005590.pub3.

17. lida T, Uchida K, Lokman N, Furukawa A, Suzuki Y, Kumasaka T, et al. Calcified granulomatous lung lesions contain abundant Mycobacterium tuberculosis components. J Mycobac Dis. 2014; doi:10.4172/2161-1068.1000142.

18. Kwon HH, Suh DH. Recent progress in the research about Propionibacterium acnes strain diversity and acne: pathogen or bystander? Int J Dermatol. 2016;55:1196-1204.

19. Walsh TR, Efthimiou J, Dreno B. Systematic review of antibiotic resistance in acne: an increasing topical and oral threat. Lancet Infect Dis. 2016; doi:10.1016/S1473-3099(15)00527-7. 
20. Nagata K, Eishi Y, Uchida K, Yoneda K, Hatanaka H, Yasuhara T, et al. Immunohistochemical detection of Propionibacterium acnes in the retinal granulomas in patients with ocular sarcoidosis. Sci Rep. 2017; doi:10.1038/s41598-017-15710-0.

21. Eishi Y. Etiologic aspect of sarcoidosis as an allergic endogenous infection caused by Propionibacterium acnes. Biomed Res Int. 2013; doi:10.1155/2013/935289.

22. Wylie KM, Blanco-Guzman M, Wylie TN, Lawrence SJ, Ghobadi A, DiPersio JF, et al. High-throughput sequencing of cerebrospinal fluid for diagnosis of chronic Propionibacterium acnes meningitis in an allogeneic stem cell transplant recipient. Transpl Infect Dis. 2016;18:227-33.

23. Brook I. Anaerobic bacteria in upper respiratory tract and head and neck infections: microbiology and treatment. Anaerobe. 2012;18:214-20.

24. Fulghum RS, Daniel HJ 3rd, Yarborough JG. Anaerobic bacteria in otitis media. Ann Otol Rhinol Laryngol. 1977;86:196-203.

25. Yang G, Eishi Y, Raza A, Rojas H, Achiriloaie A, De Los Reyes K, et al. Propionibacterium acnesassociated neurosarcoidosis: A case report with review of the literature. Neuropathology. 2017; doi:10.1111/neup.12411.

26. Okada M, Suemori K, Takagi D Teraoka M, Yamada H, Hato N. Comparison of Localized and Systemic Otitis Media With ANCA-Associated Vasculitis. Otol Neurotol. 2017; doi:10.1097/MAO.0000000000001563.

\section{Figures}

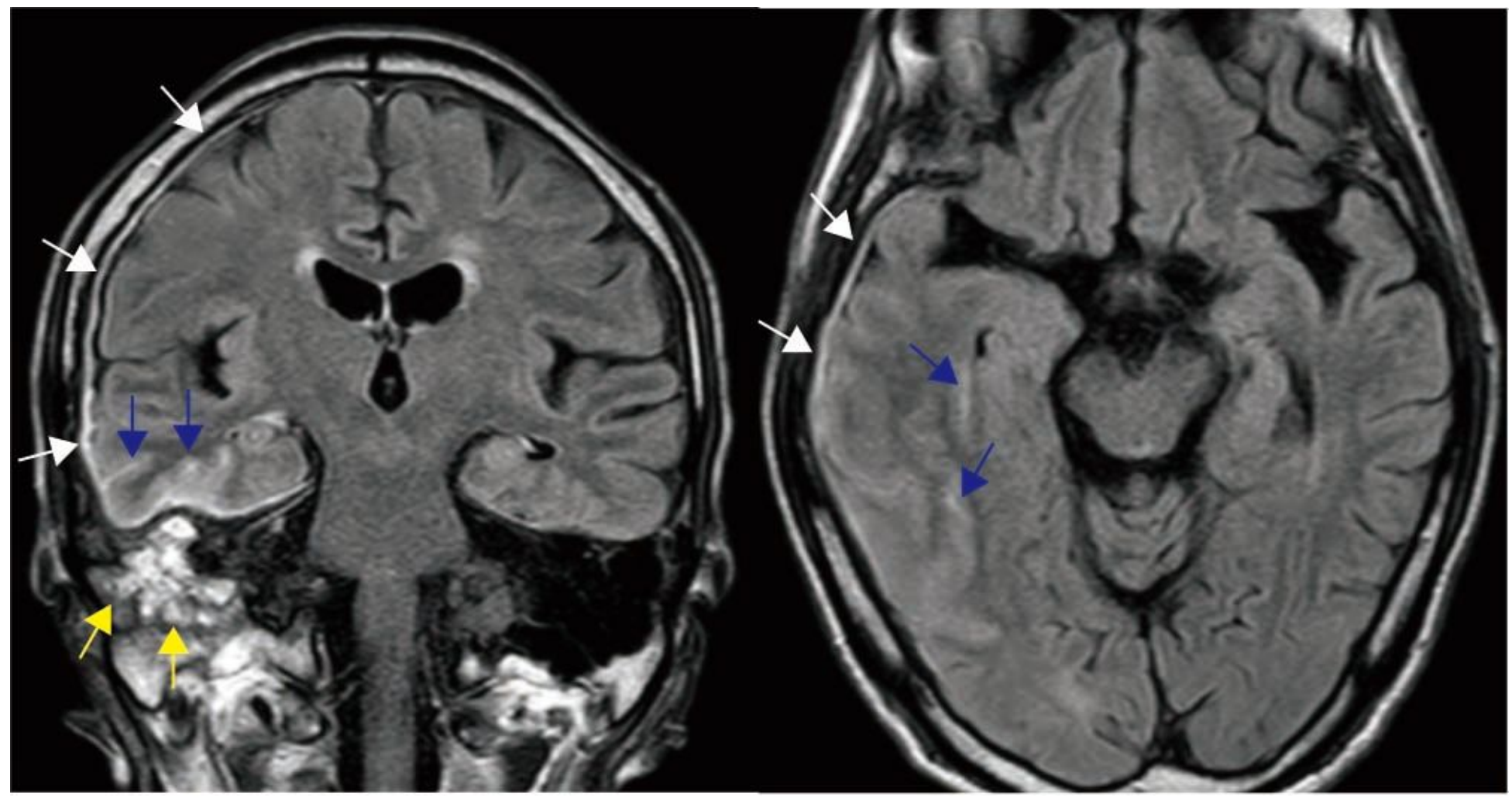

Figure 1 
Hypertrophic pachymeningitis and meningitis - Post-contrast fluid-attenuated inversioin recovery (FLAIR) on MRI, coronal section (left) and axial section (right). Gadolinium enhancement of the dura mater in the right temporal lobe and the middle cranial fossa (white arrows) and enhancement of the pia mater (blue arrows) represent hypertrophic pachymeningitis and meningitis, respectively. Right mastoiditis was also evident (yellow arrows).

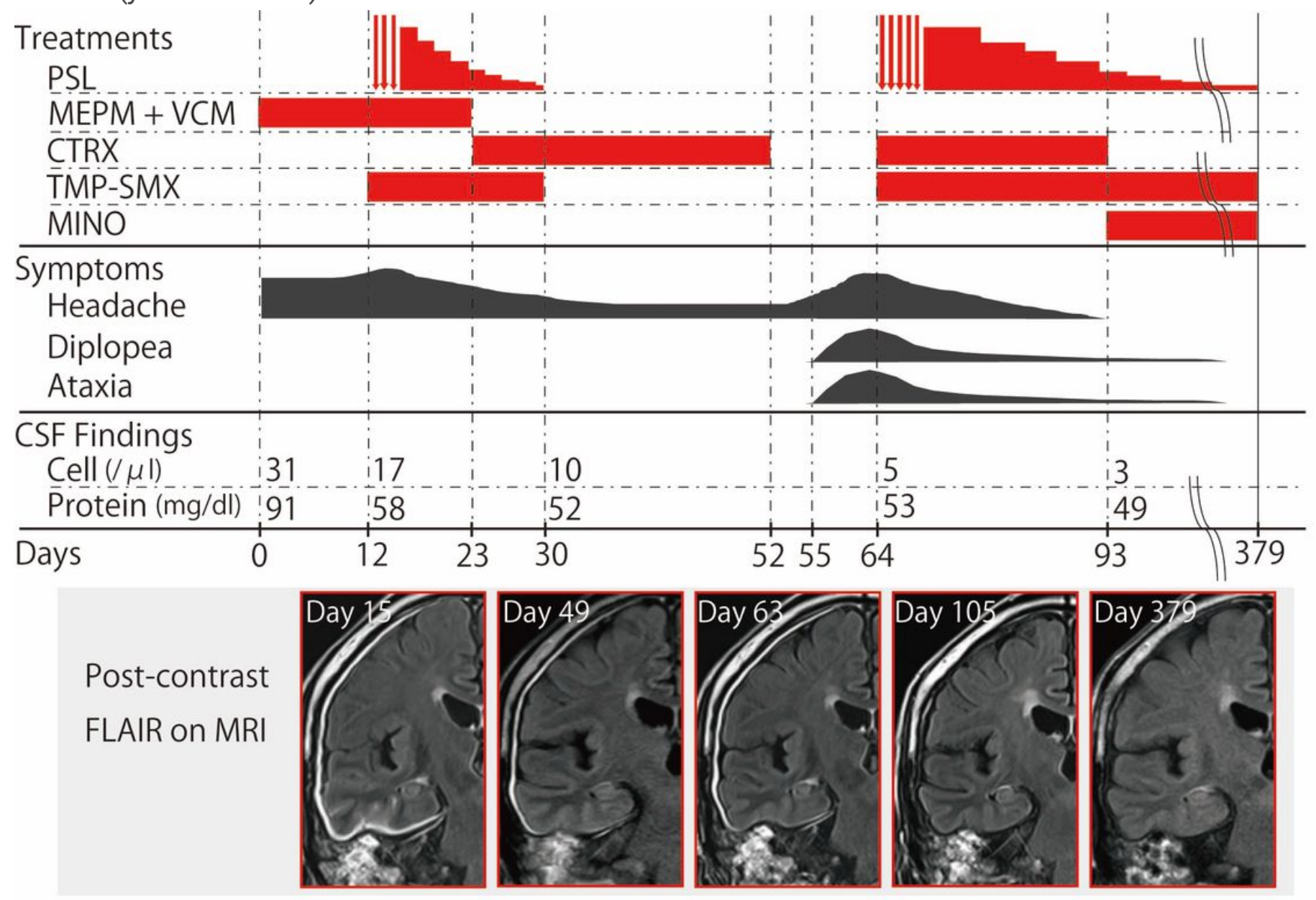

\section{Figure 2}

An overview of treatments, symptoms, CSF findings, and radiological findings on MRI - PSL: predonisolone (red arrows in treatments indicate high-dose IVMP), MEPM: meropenem, VCM: vancomycin, TMP-SMX: trimethoprim-sulfamethoxazole, MINO: minocycline, CTRX: ceftriaxone. Postcontrast FLAIR on MRI revealed that gadolinium enhancement of the dura mater, pia mater, and mastoid was diminished after the long-term administration of PSL, TMP-SMX, and MINO. 


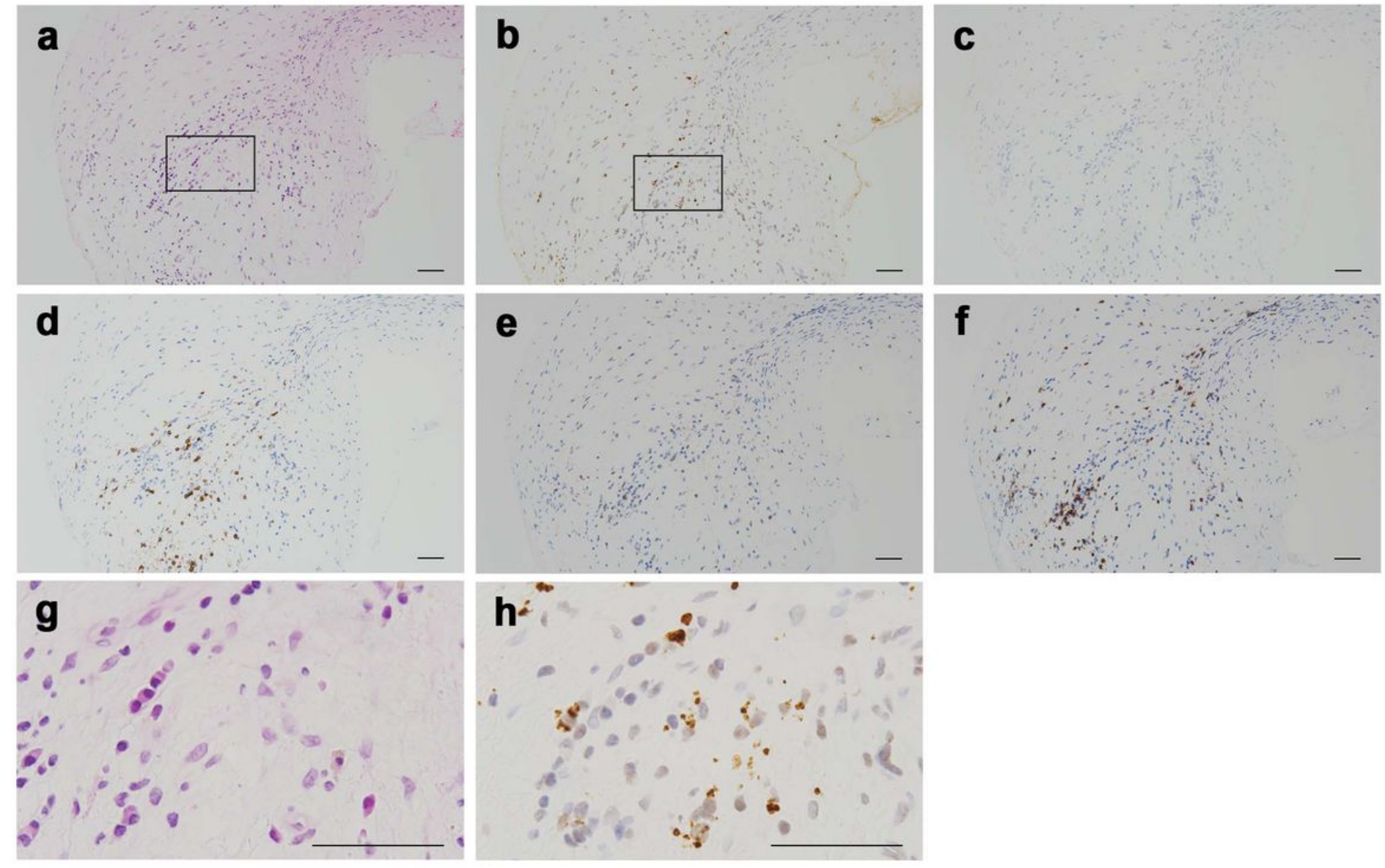

Figure 3

A biopsied mastoid tissue - (a) HE. (b) Propionibacterium acnes stain. (c) Mycobacteria stain. (d) CD20 stain. (e) CD4 stain. (f) CD8 stain. (g, h) Enlarged views of the HE and Propionibacterium stains, which are enclosed in the squares in (a) and (b), respectively. Bars: $50 \mu \mathrm{m}$. 

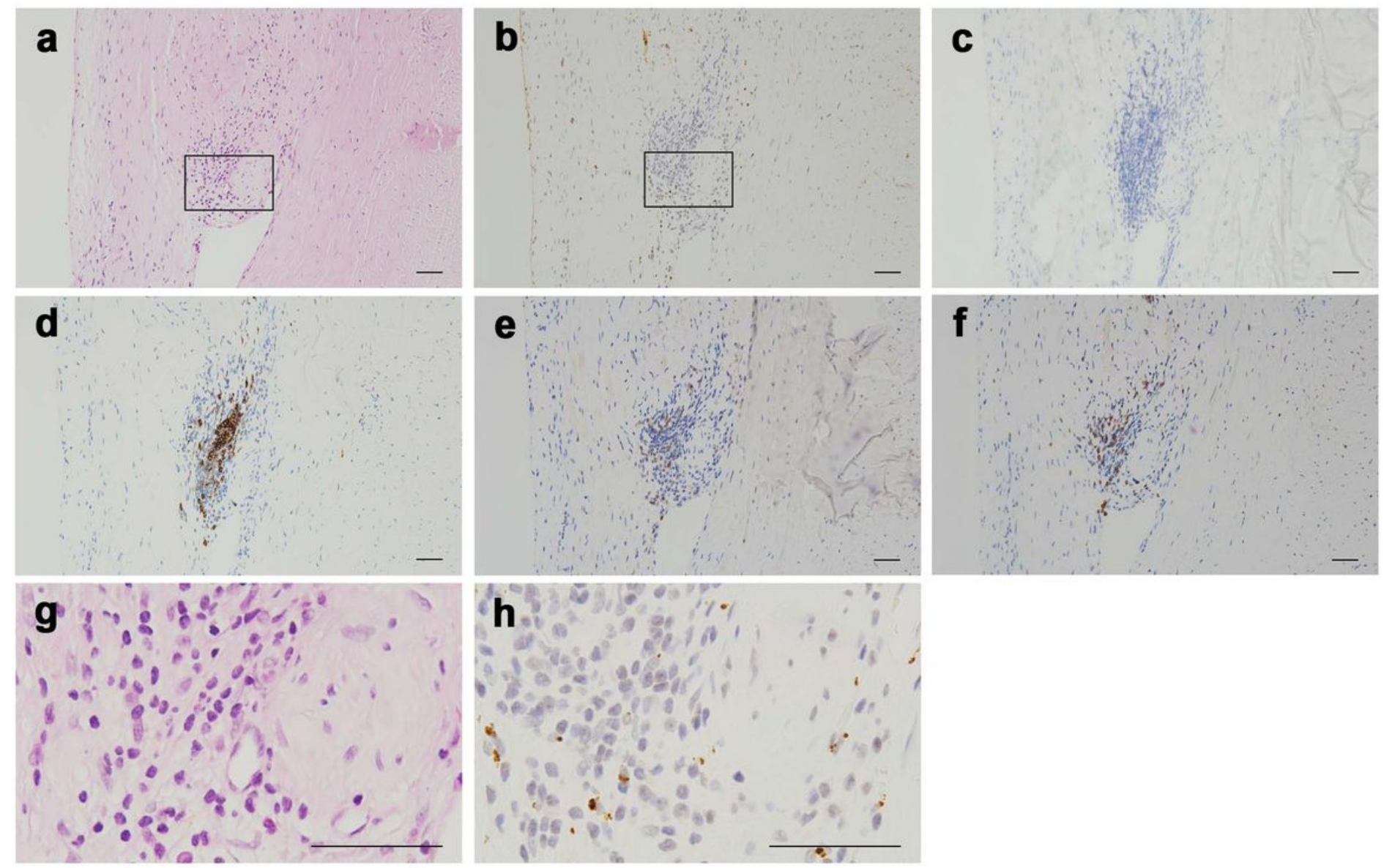

\section{Figure 4}

A biopsied tissue from the dura mater - (a) HE. (b) Propionibacterium acnes stain. (c) Mycobacteria stain. (d) CD20 stain. (e) CD4 stain. (f) CD8 stain. (g, h) Enlarged views of the HE and Propionibacterium acnes stains, which are enclosed in the squares in (a) and (b), respectively. Bars: $50 \mu \mathrm{m}$.

\section{Supplementary Files}

This is a list of supplementary files associated with this preprint. Click to download.

- supplement1.pdf 